\title{
Efficient and Improved Navigation Application for Smart Devices in IoT Environment
}

\author{
Sara Humaid Saif AL Maqbali ${ }^{\mathrm{a},}$ Muhammad Sohail Hayat ${ }^{\mathrm{a}}$
}

\begin{abstract}
Smart cities are not merely the infusion of technology into a city's infrastructure, but also require citizens interacting with their urban environment in a smart and informed manner. Transportation is key aspect of smart cities, where new route-planning applications provide as connected Car applications. For example, On-Board Human-Machine Interface (HMI) Apps, smartphone apps, API development, OBD-II, and SDL. Moreover, by using proprietary mobile apps, it is possible to get the GPS coordinates of a car, trace its route, open its doors, start its engine, and turn on its auxiliary devices. In addition, to study the usage of ICT to enhance quality and performance of urban services, reduce costs and resource consumption, and engage more effectively and actively with its citizens. The IoT is enabled by the latest developments in RFID, smart sensors, communication technologies, and Internet protocols. The basic premise is to have smart sensors collaborate directly without human involvement to deliver a new class of applications. The current revolution in Internet, mobile, and machine-to-machine (M2M) technologies can be seen as the first phase of the IoT. In the coming years, the IoT is expected to bridge diverse technologies to enable new applications by connecting physical objects together in support of intelligent decision making. This paper presents an overview of technical details that pertain to the IoT enabling technologies and applications. Compared to other survey papers in the field, our objective is to provide a more thorough summary of the most relevant application issues to enable researchers and application developers to get up to speed quickly on how ICT provide new route-planning application using IoT. We also provide an overview of some of the key IoT and M2M challenges presented in the recent literature and provide a summary of related research work. Moreover, we explore the relation between the IoT and M2M technologies. Also, present the need for better horizontal integration among IoT services. Finally, we present detailed service use-cases to illustrate how the different solutions presented in the paper fit together to deliver desired IoT \& M2M services. Since more devices are connected to the Internet every day and for various purposes, this will generate increasing amounts of data that will need to be crunched. New and better analytics systems will have to be developed, not to mention cloud storage space to deal with all the data.
\end{abstract}

Keywords: IoT, Smart devices, sensors, HMI, API, SDL, OBD-II, M2M, route planning, RFID.

\section{Introduction}

Civil governments around the world are utilizing enormous information and Internet-of-Things applications to enhance numerous parts of day by day life. Real tech organizations like IBM, Cisco and Microsoft are in on the pattern.

The Internet of Things (IoT) idea presents another and universal processing, and correspondence worldview where brilliant articles can trade data to bolster smart applications in an independent way. Without human intercession, savvy objects situated in different application areas could connect with each other to achieve many undertakings, going from social insurance to a basic ON/OFF action of a light. As indicated by the Institute of Network societies, an IoT is characterized as "a dynamic worldwide system foundation with self-designing capacities in view of standard and interoperable correspondence conventions, where physical and virtual "Things" have characters, physical properties, and virtual identities and utilize insightful interfaces, and are flawlessly coordinated into the data arrange [1].

Propels in the semiconductor, computing and communication techniques have engaged installed wireless computing to support evolutionary new networking architecture that could meet IoT prerequisites in a consistent way. It is presently apparent that in future IoT applications will be sent in all sections of the current society and industry to complete an extensive variety of exercises. Henceforth, it is basic to change the texture of the key correspondence foundation of the Internet to empower consistent correspondence among the wise gadgets. Such changes will be an outlook change from human-to-human correspondence towards astute machine-to-machine correspondence [3]. The traditional idea of the Internet as a framework organize connecting with end-client terminals will blur, offering ascend to an interconnected situation where brilliant items will utilize inescapable figuring conditions. IoT applications will spread in many existing and new ICT (Information and Communications Technology) regions including those where the Internet has not yet come to. The application necessities will in this manner essentially expand, requiring installed smart network architecture [1].

The IoT idea presents a noteworthy move in ICT regions where, rather than interfacing end-client gadgets, the physical articles will associate with each other and collaborate with anything. The idea of interconnected articles was created from the organization of RFID (Radio Frequency Identification Device) for naturally distinguishing and following items. The thought was bolstered with the development of remote sensor arranges in the mid to late 1990's the place propelled checking applications rose [2]. Implanted figuring and low power remote specialized gadgets productively bolster network among astute items. As of late, the idea of IoT has additionally progressed into the Internet of Nano Things (IoNT) [1]. The IoNT idea has been marked as frameworks where smaller than expected, conceivably detached sensors now and again, interconnected through nano-systems could get fine grain information from difficult to-get to areas, for example, the human body or inside complex machines. A portion of the main ICT organizations, for example, Cisco, IBM are currently driving the limit of the IoT to call the territory as the IoE (Internet of Everything), which is a further speculation of the IoT idea 
Application areas of the IoT or the IoNT are not restricted by current-day applications just; they will develop as the empowering bolster framework moves forward. The IoT will be an empowering agent to numerous application spaces, including inventory network administration, transport and coordinations, assembling, aviation and car, medicinal services, security and wellbeing, social administrations, and so forth. As per experts, 50 to 100 billion gadgets will be associated by the Internet by the year 2020 (Mobile world). The worldwide market for sensors is required to increment to US $\$ 91.5$ billion by 2016, speaking to a compound yearly development rate of 7.8\% [4]. The IoT application areas will come in various sizes and shapes that create little to enormous measure of information. For instance, a Boeing plane could produce $10 \mathrm{~TB}$ (terabytes) of information per motor like clockwork. In an airplane, despite the fact that the physical size of a system could be little, the quantity of information generators could be gigantic.

Internet of things is a sophisticated concept of the Internet so that possesses all things in our ability to contact each other or the Internet to send and receive data to perform specific functions through the network. By 2020, the size of the Internet market will be greater than the market for mobile phones, computers and tablets combined by twice as much! Where the number of Internet devices will reach 35 billion devices connected to the Internet.

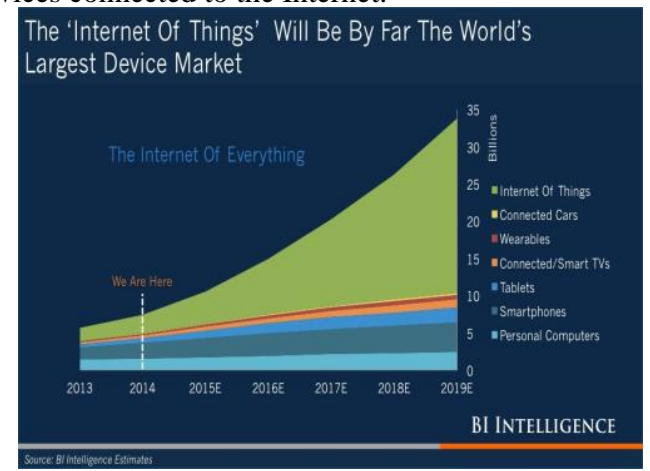

Figure 1: IoT Development [1]

\section{IoT applications \& systems}

In 1999 Kevin Ashton initially proposed the idea of the Internet of Things (IoT) that immediately picked up ubiquity among analysts and different specialized gatherings [11]. The IoT idea came into the mechanical spotlight when the International Telecommunications Union (ITU) distributed the main give an account of IoT in 2005. From that point forward, the idea of IoT has been grasped by specialists, different guidelines bodies, for example, the Institute of Electrical and Electronic Engineers (IEEE), the European Telecommunication Standards Institute (ETSI), the Internet Engineering Task Force (IETF), the ITU and numerous other national and worldwide associations.

As of late, numerous universal research programs have been started, e.g., EU structure FP7 program, National Institute of Standards and Technology and National Science Foundation (NIST) in the USA. Fast improvement of lowpower gadgets, correspondence advances and information scientific procedures permitted far reaching sending of IoT applications and frameworks.

As indicated by Gartner, a main worldwide Information Technology investigate organization, the quantity of introduced IoT associated gadgets will achieve 26 billion by
2020, up from 0.9 billion in 2009[10]. As indicated by Cisco, the quantity of web associated gadgets has achieved 8.7 billion in 2012 [9]. Associated IoT gadgets are using the machine to machine (M2M) correspondence design for the availability. The original of IoT applications depended on the Radio Frequency Identification (RFID) innovation for coordinations, retail and comparative different parts. The IoT applications later incorporated with distributed computing and remote sensor organize engineering. The principle parts of an IoT framework are savvy gadgets, circulated registering abilities and consistent interchanges capacities as appeared in figure. A shrewd question can be portrayed by the accompanying qualities [8].

- It has an interesting personality, which is addressable by different self-governing gadgets.

- The question can self-governingly interface with different gadgets and can execute certain assignments.

- It has processing abilities to fulfill certain assignments either in an independent or in a helpful way.

- It might have a few capacities to detect encompassing physical situations as well as wonders or can trigger certain procedures in light of its detecting abilities. The activated procedures either could dwell inside itself or could exist in a dispersed manner.

- A portion of the articles may need to meandering abilities supporting versatile applications.

The above general qualities could be utilized as a part of various IoT applications going from straightforward applications, for example, sensorconstruct light In light ON/OFF to control of selfsufficient associated vehicles on open streets to enhance the unwavering quality of future transportation frameworks. Showcase segments and application ranges of the IoT are very vast and will extend with time. It is impractical to depict all ranges; be that as it may, here we can aggregate these applications into various significant classifications and describe the regions as far as their correspondence needs. IoT application and market segments can be assembled into taking after seven classes [3]. Each of the classifications of uses is summarily depicted underneath.

- Smart home/structures

- Smart City

- $\quad$ E-Health and therapeutic frameworks

- $\quad$ Street movement and transportation frameworks

- Environmental management

- Business stock and item administration

- Industrial automation and manufacturing

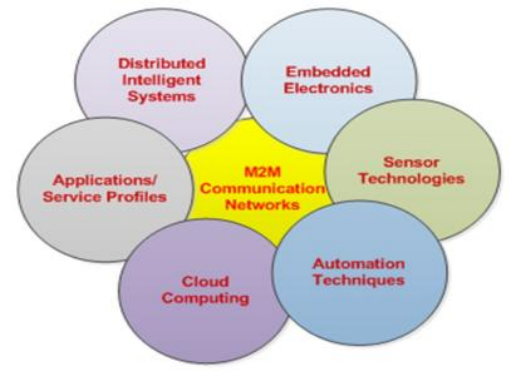


Figure 2: Basic building blocks of IoT Systems [1]

Smart home/structures: IoT gadgets and network could be sent in homes and additionally in huge structures. Sensor gadgets can be introduced inside homes or structures to actualize vitality effectiveness, security and wellbeing applications, machine control utilizing home region systems (HAN) and numerous different applications [7]. The vast majority of the applications are detecting and checking; however new applications are developing to encouraging group of people associated gadgets as can be found in a smart matrix condition. Some propelled keen home machines could utilize network to get privately produced data from on location sustainable power source generators to plan the operation of apparatuses. A large portion of the applications in this condition will work in either neighborhood individual territory organizing (LAN/PAN) situations with short transmission remove between hubs. Machines inside a HAN could utilize the smart meter as the entryway/switch for associations with actualize request administration framework by trading information with the power organization server.

Smart City: A smart_city based IoT framework will incorporate numerous utility administrations, where numerous suppliers could offer propelled administrations. This condition can be viewed as a digital physical eco-framework where best in class correspondence and figuring foundation will be important to bolster administrations. Benefits in this condition could incorporate street activity control, stopping organization, person on foot wellbeing; general wellbeing and security, fundamental administrations, for example, power, water and different administrations administration, road lighting control, and so on. Smart_cities could support fragmented IoT services where different service providers could have their own real infrastructure or use some form of virtual infrastructure by sharing common resources.

Applications in these situations could have multi-level QoS prerequisites, which may force numerous confinements on the foundation outline. For instance, some security applications could trigger numerous sensors requiring low inactivity information exchange abilities from its system and in addition high transmission transfer speed to transmit video. Correspondingly a power lattice control framework may require a low inertness transmission interface if there should be an occurrence of a blame created inside a network yet may not require high transmission data transfer capacity.

One of the basic issues in a smart city IoT condition is additionally the sheer number of gadgets that should be adjusted. From the correspondence organizing perspective, both customary customer server design and gadget to-gadget interchanges bolster should be given. The smart city IoT condition could be very unpredictable; especially on the grounds that in future it might be conceivable to give numerous community offices in associated modes. For instance, different government offices and organizations in the USA and different nations are thinking about the advancement of associated auto frameworks which may lessen city parking spot necessities by transport developments in light of the worker's prerequisites as opposed to where right now suburbanites arrange their errands in light of transport calendars. This could be an enormous development zone that requires noteworthy innovative work push to grow new foundation.

Another noteworthy zone inside a smart city is the security and reconnaissance framework. IoT empowered gadgets could essentially upgrade the present system of surveillance cameras to find interlopers or guilty parties. Arranged security sensors and robotized cameras could work out the development ways of gatecrashers empowering security administrations to act all the more rapidly. For instance, costly things could be labeled with dynamic radio transmitters that could radiate mark signals while stolen products are being brought through the work of sensors situated in structures and on streets/pathways. Such IoT applications could be a piece of future civil or city systems. A shrewd city arrange should be executed in light of cutting edge heterogeneous system design.

\section{Road traffic and transportation systems:}

Road traffic systems are as of now utilizing essential ICT frameworks, for example, programmed movement signals, online activity checking, Global Positioning System (GPS) based direction frameworks, open transport planning frameworks, in system control frameworks, and so forth. Notwithstanding, this region could colossally profit by the utilization of IoT/M2M correspondence frameworks. As of late critical $R \& D$ interests have been produced in the car part for associated and self-governing autos where vehicles can trade data either among themselves or with different elements to upgrade and enhance course direction frameworks, offer improve transportation benefits and enhance walker wellbeing [4].

In order to support the connected car environments huge research advancement exercises have begun including the VANET (Vehicular Ad hoc Network) gauges in view of IEEE 802.11p and Dedicated Short Range Communication (DSRC) measures. Associated auto frameworks could be reached out to enhance add up to street activity frameworks wellbeing by interfacing with people on foot through their advanced mobile phones and furthermore with prepare frameworks to keep away from accidents at rail intersections. Such frameworks should be absolutely self-ruling and ought to be capable bolster information trade between various substances with a high level of certainty. To maintain a strategic distance from auto/passerby impacts, Honda is currently trialing VANET based crash shirking frameworks in USA in conjunction with the Qualcom [5]. The utilization of IoT based frameworks for the vehicle segment will improve the unwavering quality and wellbeing of street activity frameworks and additionally permitting offering new methods of open transport frameworks. To bolster vehicular IoT frameworks it is important to take a shot at vehicular industry standard based frameworks.

\section{Business inventory and product management}

RFID technologies are altogether sent in numerous business areas for stock administration, all through the supply and stock administration. The RFID based sensors enables the frameworks to distinguish items and give them following capacities. Such RFID based frameworks are utilized as a part of many structures, in-house to track item stock and also between site developments utilizing nearby and wide range systems. For instance, as of late satellite based M2M frameworks are getting to be plainly prominent for stock and store network administration with a specific end goal to find and screen the development of delivery compartments crosswise over areas or a nation and in addition for crosscountry developments. Various satellite M2M specialist 
organizations offer propelled administrations that incorporate ORBCOMM, Iridium, Globalstar, Inmarsat and few others (M2M Satellite). For future applications other sort of sensors, incorporating biosensors in blend with RFID advancements could permit control of generation procedures, enhancing the nature of item and time span of usability, and opportune conveyance of items.

\section{COMMUNICATION NeTWORKS FOR FUTURE IOT SySTEMS}

The communication network is one of the key building squares of the IoT and distributed computing frameworks. As specified in the past segment, the IoT idea has been produced in view of the $\mathrm{M} 2 \mathrm{M}$ correspondence engineering. For achievement of IoT ventures it is important to build up an open industry standard that is merchant free and can without much of a stretch interwork with other utilitarian modules. Figure 2 demonstrates a useful model of the M2M correspondence organize in view of the ETSI (European Telecommunications Standards Institute) display (ETSI TS102 690). Figure demonstrates that the system is partitioned into three layers: zone, get to and center systems. The territory system is near the IoT gadgets giving direct availability to these gadgets. It is by and large expected that these gadgets will work at low power level, consequently a system association is fundamental in the region of these essential IoT gadgets. It is in all likelihood that the majority of the IoT gadgets will utilize remote availability to interface with a region arrange.

Next level in the system pecking order is the get to network that associates a few region arranges and also some upgraded IoT gadgets to think information. The get to organize additionally associates with the M2M passage gadgets that think numerous M2M capacities and abilities. The greater part of the M2M benefit abilities and applications will be conveyed over a substantial range associated by means of the center system. Correspondence between gadgets should be offered at various levels to actualize numerous IoT applications. For instance, a sensor in a range system may actuate a switch in the same or distinctive territory organize. On the off chance that the sensor and switch are in a similar region arrange then it might essential for relating applications to give gadget to gadget availability. Henceforth, new gadget to gadget and conventional customer server availability models must be kept up inside the M2M correspondence design for the IoT applications

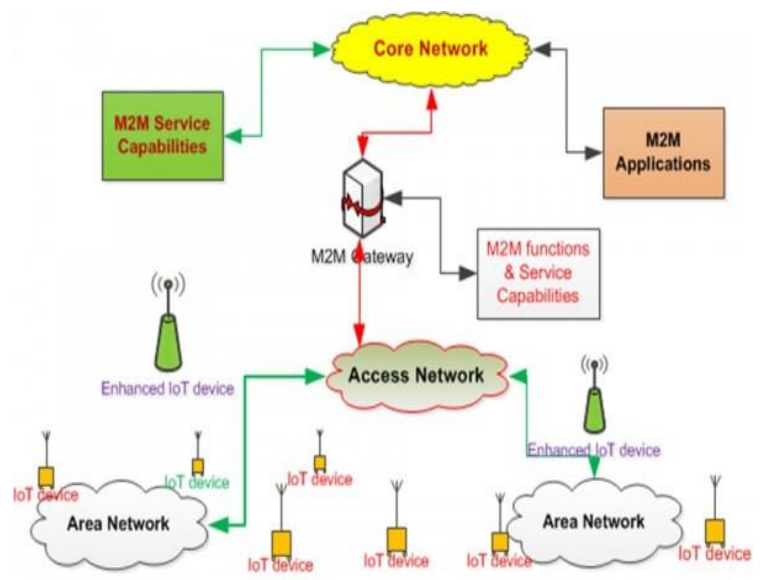

Figure 3: Machine to machine communication architecture

Considering the IoT classifications talked about in Section II and the M2M correspondence design, it will be important to utilize various interchanges advancements to give network to scope of IoT applications. These correspondence gauges are extensively arranged either as wired or remote correspondence frameworks. Wired correspondence frameworks additionally incorporate fiber-based optical systems. A portion of the present key correspondence arrange benchmarks that could be utilized for IoT interchanges are recorded in Table 1 [5]. The majority of the systems are multi-benefit systems i.e. these systems can serve various sorts of movement. A portion of the remote systems administration norms which are more proper for IoT applications are depicted later

Table 1: Summary of communication networking technologies

\begin{tabular}{|c|c|c|c|}
\hline $\begin{array}{l}\text { Network } \\
\text { Type }\end{array}$ & $\begin{array}{l}\text { Network } \\
\text { Size }\end{array}$ & Standard & Features \\
\hline \multirow[t]{7}{*}{ Wired } & \multirow[t]{3}{*}{ WAN } & \multirow[t]{3}{*}{ DSL, FTTN ot FTTH } & DSL: $2-20 \mathrm{Mbits} / \mathrm{sec}$ \\
\hline & & & FTTN/FTTH: 2 -1000Mbits/sec \\
\hline & & & Both are infrastructure based standards \\
\hline & \multirow[t]{4}{*}{ LAN } & \multirow[t]{3}{*}{ Ethemet 802.3} & Ethernet: $10 \mathrm{Mbits} / \mathrm{sec}$ \\
\hline & & & Fast Ethernet: $100 \mathrm{Mbits} / \mathrm{sec}$ \\
\hline & & & Gigabit Ethernet: $1,10 \mathrm{Gbits} / \mathrm{sec}$ \\
\hline & & Homeplug: HomePNA/IEEE1901 & $14200 \mathrm{Mbits} / \mathrm{sec}$ \\
\hline \multirow[t]{12}{*}{ Wireless } & \multirow[t]{7}{*}{ WAN } & \multirow[t]{3}{*}{ 2G: GSM/GPRS/EGPRS } & GSM: upto $57.6 \mathrm{Kbits} / \mathrm{sec}$ \\
\hline & & & GPRS: $56-114 \mathrm{Kbits} / \mathrm{sec}$ \\
\hline & & & EGPRS: upto $236 \mathrm{Kbits} / \mathrm{sec}$ \\
\hline & & 3G:UMTS & upto $2 \mathrm{Mbits} / \mathrm{sec}$ \\
\hline & & 3.5G: HSPA, & upto $42 \mathrm{Mbits} / \mathrm{sec}$ \\
\hline & & WIMAX & upto $70 \mathrm{M} \mathrm{bits} / \mathrm{sec}$ \\
\hline & & 4G: LTE & upto $100 \mathrm{Mbits} / \mathrm{sec}$ \\
\hline & LAN & WLAN & $2-400 \mathrm{Mbits} / \mathrm{sec}$ \\
\hline & \multirow[t]{4}{*}{ PAN } & Bluetooth & $1-10 \mathrm{Mbits} / \mathrm{sec}$ \\
\hline & & Zigbee & $250 \mathrm{Kbits} / \mathrm{sec}$ \\
\hline & & Ultra Wideband & upto $480 \mathrm{Mbits} / \mathrm{sec}$ \\
\hline & & ISA100.11a & $250 \mathrm{Kbits} / \mathrm{sec}$ \\
\hline \multirow[t]{2}{*}{ Satellite } & \multirow[t]{2}{*}{ WAN } & \multirow[t]{2}{*}{ LEO: Low Earth Orbit Satellite } & $\begin{array}{l}160-2000 \mathrm{~km} \text { height, constellation of } 25 \\
\text { satellites }\end{array}$ \\
\hline & & & $50 \mathrm{Mbits} / \mathrm{sec}$ \\
\hline
\end{tabular}

GSM: Global System for Mobile, GPRS: GSM Packet Radio System, EGPRS: Enhanced GPRS, UMTS: Universal Mobile Telecommunications System, HSPA: High Speed Packet Access, WiMAX: Worldwide Interoperability for Microwave Access, LTE: Long Term Evolution, WLAN: Wireless Local Area Network, 6LoWPAN: IPv6 Low Power Wireless Personal Area Network.

Essential prerequisites of IoT Networks: Before creating system design it is imperative to comprehend essential IoT organizing necessities. IoT gadgets and applications will create information from different sensors and electronic gadgets which are by and large short information blasts of a couple of bits to most extreme of several hundred bits, producing either in a synchronous or nonconcurrent form. The greater part of the IoT gadgets working in zone systems ought to work in a low power condition so that sensor hubs battery or the vitality source could keep going for long time. Visit 
vitality source substitution could be the fundamental obstruction towards the mass sending of these gadgets and applications.

\section{Conclusion}

This paper introduced an extensive audit of the IoT applications and correspondence organize plan methods. The paper checked on various correspondence advancements appropriate for IoT applications. The paper demonstrated that both remote and wired systems could be utilized to create future IoT frameworks; nonetheless, because of the way of the framework, remote advancements will overwhelm the systems administration region. IoT is changing our life. Machine learning changes the machine comprehension to human, with the capacity managing Big Data, those applications are getting to be plainly basic to our life, also pursued by Venture Capitals. Then again, those application simply can make the expectation in light of the past history, what's to come is still in some way or another unverifiable, that is the enchantment

\section{References}

Akyildiz (2010) 'The Internet of Nano-Things', IEEE Wireless Communications, pp. 58-63.

Atzori, L; Iera, A; Morabito, G (2011) 'The Internet of Things: A Survey', Computer Networks, pp. 27872805.

Balasubramaniam, S;Kangasharju, J (2013) 'Realizing
Internet of Nano Things: Challenges, Solutions and Applications', IEEE Computer, pp. 62-68.

Chen, D; Khan, J.Y; Brown, J (2014) 'An Area Packet Scheduler to Mitigate the Coexistence Issue in a WPAN/WLAN Based Heterogeneous Network', IEEE WCNC 2014, pp. 2-9.

Iniewski, K. (2015) 'Convergence of Mobile \& Stationary Next-Generation Networks', IoT, pp. 57-68.

Jamil Y. Khan , [Online].

Jamil Y. Khan; Dong Chen; Oliver Hulin (2014) Enabling Technologies for Effective Deployment of Internet of Things (IoT) Systems, 4 December, [Online], Available: HYPERLINK "http://telsoc.org/ajtde/2014-12-v2-n4" http://telsoc.org/ajtde/2014-12-v2-n4 .

Kim, J; Lee, J; Yun, J. (2014) 'M2M Service Platforms: Survey, Issues and Enabling Technologies', IEEE Communications Surveys \& Tutorials, pp. 61-76.

Miorandi, D; Sicari, S; De Pellegrini, F; Chlamtac, I (2012) Internet of things: Vision, applications and research challenges". NewYork : 1497-1516

Sterling, L; Tareter, K (2010) Intelligent Lifestyle Applications. MIT Press : 1st edition

Yuce M; Khan, J (2013) 'Wireless Body Area Networks: Technology, Implementation, and Applications', Pan Stanford Publishing, pp. 14-16.

Zaslavsky (2013) 'Internet of Things and Ubiquitous Sensing', Computing Now, pp. 5-6. 